\title{
Primary Pulmonary Epithelioid Hemangioendothelioma: A Case Report and Literature Review
}

Ambrosetti $\mathrm{T}^{1}$, Sgardello Sébastian $\mathrm{D}^{2}$, Thomopoulos $\mathrm{T}^{2}$, Koliakos $\mathrm{E}^{1}$, Strano $\mathrm{F}^{1}$, Abdou $\mathbf{M}^{3}$, Christodoulou $\mathbf{M}^{1}$ and Abbassi $\mathbf{Z}^{\star}$

${ }^{1}$ Department of Visceral Surgery and Transplantation, University Hospital of Geneva, HUG, Geneva, Switzerland

${ }^{2}$ Department of General Surgery, Hospital of Valais, Valais, Switzerland

${ }^{3}$ Department of Anatomopathology, Hospital of Sion, Valais, Switzerland

\begin{abstract}
Introduction: Pulmonary epithelioid hemangioendothelioma (P-EHE) is a rare vascular tumour of borderline malignancy with a clinical course that lies between a benign hemangioma and an angiosarcoma. The aetiology of P-EHE is still unknown.
\end{abstract}

Method: We report a case of an incidental diagnosis of P-EHE in a 54-year-old female with multiple bilateral nodules discovered after a routine chest $x$-ray following a 1 month history of cough, dyspnea and persistent fatigue diagnosed with a right wedge resection.

Discussion: Clinical presentation is heterogeneous, with the majority of patients being asymptomatic at the time of diagnosis. A diagnosis is usually made after the discovery of abnormal findings in chest x-rays during routine health examinations. To this day, there is no approved standardised treatment protocol for P-EHE. Therapeutic options range from regular follow-ups with no active therapy in asymptomatic patients, to surgical resections, corticosteroids, azathioprine and combination chemotherapy in symptomatic persons.

Reports on survival vary greatly. Three separate studies have shown a mean survival of 4.6 years with a range of 6 months to 24 years.

Conclusion: P-EHE is a rare tumour that requires further research to correlate its radiological findings to its molecular and genetic characteristics in order to assess their prognostic value and devise standardised treatment protocols.

\section{Introduction}

The term hemangioendothelioma, first introduced by Weiss in 1986 [1], refers to a large spectrum of vascular tumours with an epithelioid and histiocytoid appearance that presents intermediate histological and clinical characteristics considered to be in between malignant angiosarcomas and benign hemangiomas. The most common forms in this group are epithelioid hemangioendotheliomas (EHE) and are typically found in soft tissue of adults around medium to large calibre veins.

Dail and Liebow originally referred to pulmonary epithelioid hemangioendothelioma (P-EHE) as an intravascular bronchioloalveolar tumour [2]. In 2004 the World Health Organisation (WHO) classified P-EHEs as low to intermediate grade vascular neoplasms because through the use of immunohistochemical techniques, Corrin et al. demonstrated the presence of tumour cells deriving from a lineage that could differentiate along endothelial lines $[3,4]$.

The aim of our paper is to report an incidental discovery of multiple, bilateral P-EHE diagnosed after a left pulmonary wedge resection.

\section{Case Report}

A 54-year-old female with a 1 month history of a chronic, nonproductive cough with persistent fatigue and a NYHA stage II dyspnoea had a chest radiograph that showed 4 bilateral lung nodules ranging between 6, 7 to 9, $6 \mathrm{~mm}$ in diameter (Figure 1). A follow-up chest CT scan showed a total of 9 separate nodules with distribution in both lungs and with sizes ranging between 5 to $7 \mathrm{~mm}$ in diameter. Four nodules ranging in size between 5 to $6 \mathrm{~mm}$ were distributed in the right lung ( 4 in the middle lobe and 1 in the inferior lobe) whilst 5 nodules with sizes ranging between 5 and $7 \mathrm{~mm}$ were distributed in the left lung (2 in the upper lobe, 1 in the lingula and 2 in the inferior lobe). The largest 5 nodules are shown in Figures 2-6. No subclavian, thoracic, axillary, mediastinal or hilar adenopathies were found. A follow up chest scan performed 3 months later showed no significant progression in nodule size, and no new nodules or adenopathies were reported.

Further investigations included an abdominal CT scan, a colonoscopy and a gynaecological exam that were negative for primary lesions. Physical examination of the patient was unremarkable as were her blood tests. The patient's prior medical history revealed a Hashimoto's thyroiditis, 2 operations for a Morton's neuroma in her left foot and a cervical hernia that was treated surgically. The patient is a non-smoker with an unremarkable family history and there was no occupational exposure of clinical relevance.

Following the initial investigation, a percutaneous transthoracic lung biopsy was proposed but the procedure was unsuccessful because of technical difficulties linked to the position of the nodules. For this reason, a thoracoscopic wedge resection of the middle lobe was performed, following a CT guided marking of the lung nodules.

The pathological examination of the specimens was carried out at the Institut Central des Hôpitaux Valaisains (ICHV) of Sion Hospital (Switzerland) where the diagnosis of P-EHE was made. The Institut Universitaire de Pathologie of the Centre Hospitalier Universitaire Vaudois (CHUV) of Lausanne Hospital (Switzerland) carried out a second analysis that confirmed the diagnosis.

*Corresponding author: Ziad Abbassi, Department of General Surgery, Hospital of Valais (CHVR), Av. du Grand-Champsec 80, Case Postale 736, 1951 Sion, Switzerland Tel: +41-766931667/+41-0767048548; E-mail: Ziad.abbassi@hopitalvs.ch/ SebastienDouglas.Sgardello@hopitalvs.ch

Received June 06, 2018; Accepted July 09, 2018; Published July 16, 2018

Citation: Ambrosetti T, Sgardello Sébastian D, Thomopoulos T, Koliakos E, Strano F, et al. (2018) Primary Pulmonary Epithelioid Hemangioendothelioma: A Case Report and Literature Review. Lung Dis Treat 4: 127. doi:10.4172/2472-1018.1000127

Copyright: @ 2018 Ambrosetti T, et al. This is an open-access article distributed under the terms of the Creative Commons Attribution License, which permits unrestricted use, distribution, and reproduction in any medium, provided the original author and source are credited. 


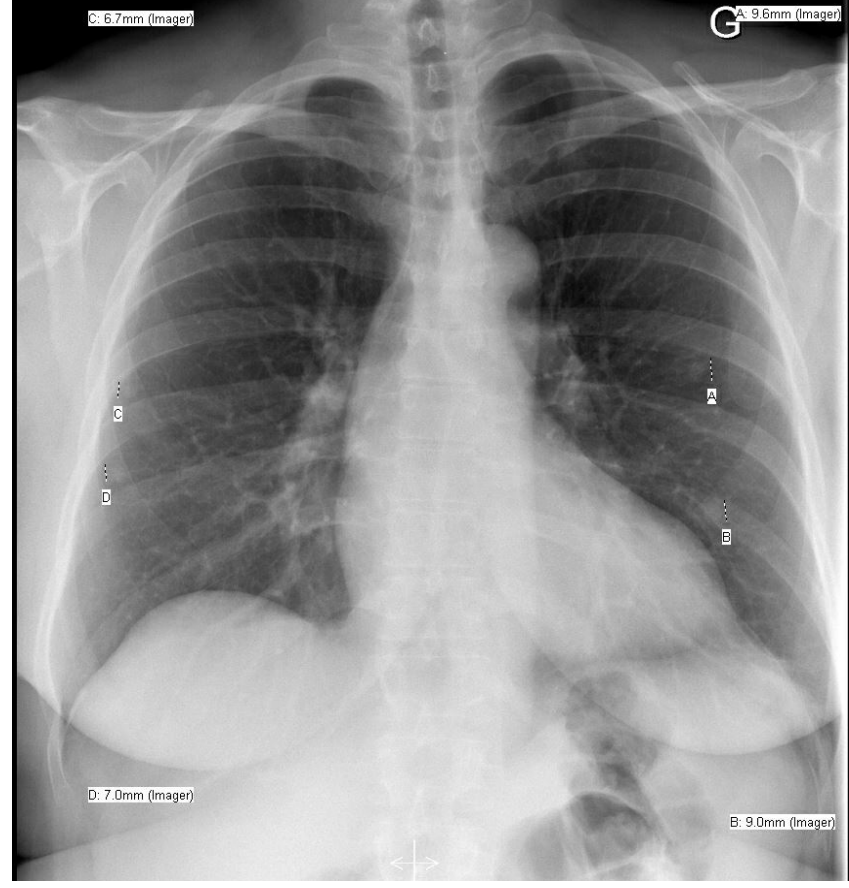

Figure 1: Initial chest x-ray showing 4 bilateral lung nodules.

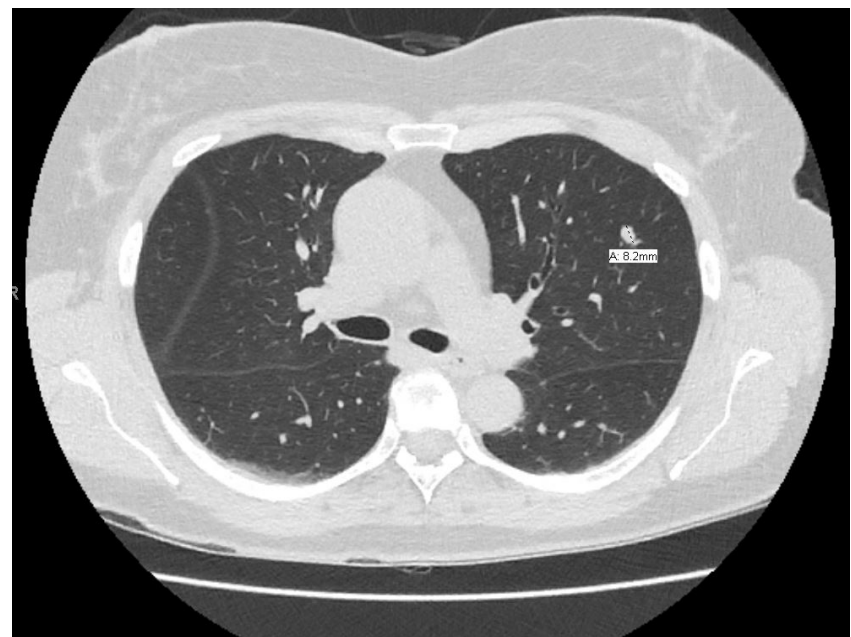

Figure 2: Chest CT scan showing a left superior lobe nodule measuring 8.2 $\mathrm{mm}$ in diameter.

The nodules were relatively well defined and lobulated with round to oval epithelial/epitheliod cells with a hyaline stroma (Figures 7 and 8 ). The cytoplasm was eosinophilic and showed vascular spaces. In certain areas, the cytoplasm was clearer and contained eosinophilic vacuoles (Figures 9 and 10).

Positivity to CD31, CD34 and factor VIII confirmed the endothelial nature of the cells. Other epithelial markers, keratin and TTF1 were negative. Vimentine was partially expressed whilst there was no expression of actin and of $\mathrm{S} 100$ protein. The proliferation index assessed using MIB1 was relatively low and was measured at $5-10 \%$ in hot spots.

The operation and the postoperative course were uneventful. A magnetic resonance imaging scan performed 6 weeks after her discharge showed no secondary lesion in the head nor in the abdomen.

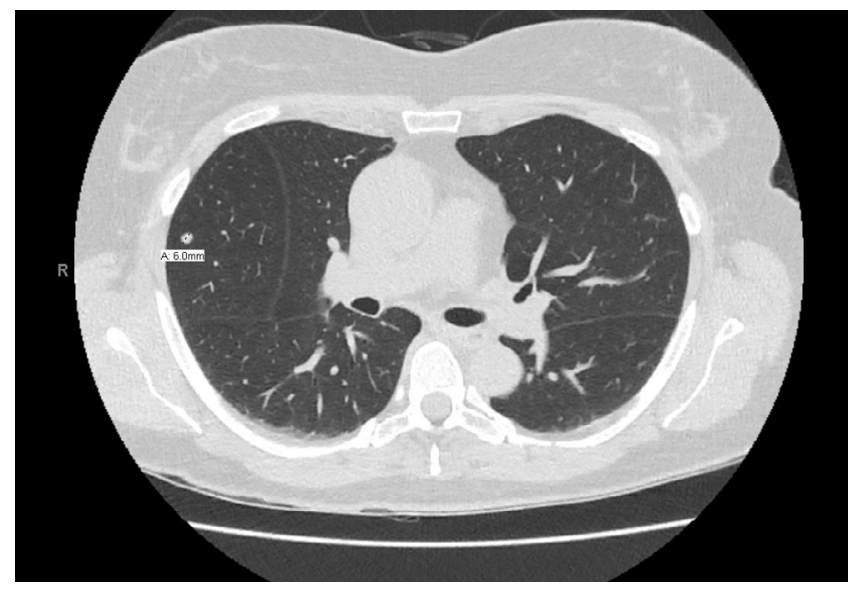

Figure 3: Chest CT scan showing a middle lobe nodule measuring $6.0 \mathrm{~mm}$ in diameter.

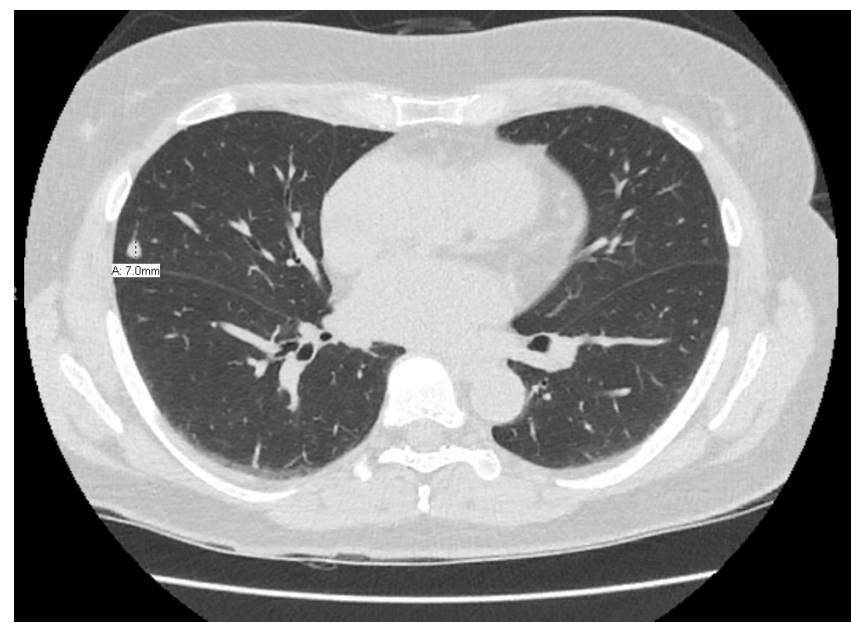

Figure 4: Chest CT scan showing a right superior lobe nodule measuring 7.0 $\mathrm{mm}$ in diameter.

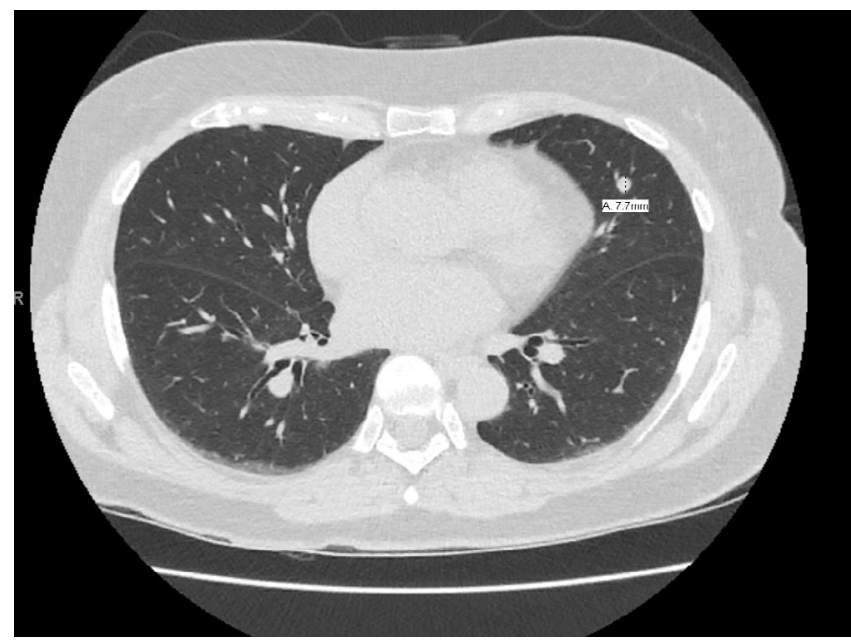

Figure 5: Chest CT scan showing a left superior lobe nodule measuring 7.7 $\mathrm{mm}$ in diameter. 


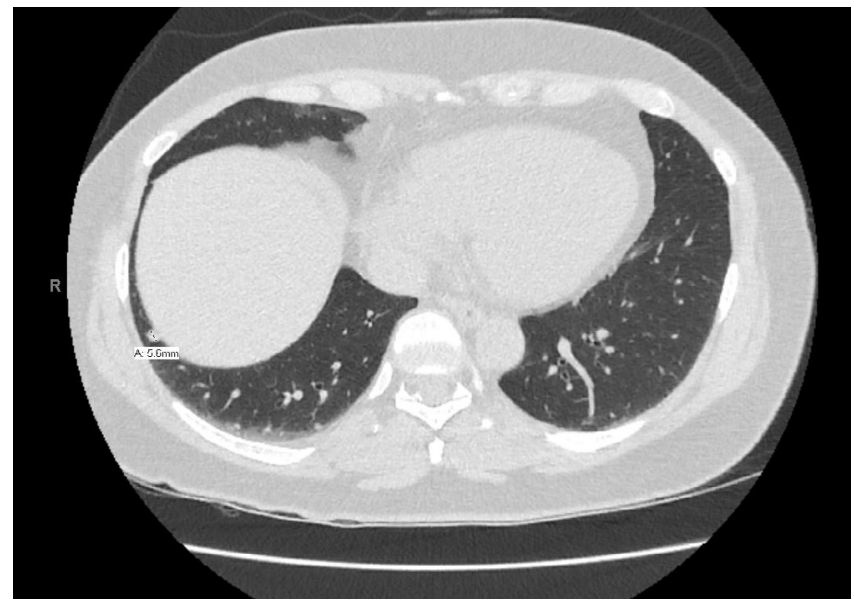

Figure 6: Chest CT scan showing a right inferior lobe nodule measuring 5.6 $\mathrm{mm}$ in diameter.

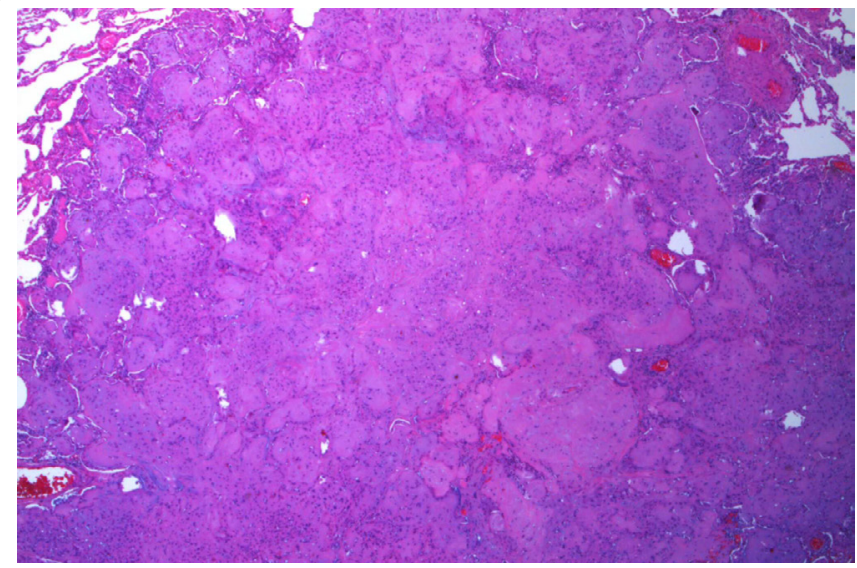

Figure 7: The tumour is well-circumscribed but not encapsulated and surrounded by lung parenchyma H\&E x2.5.

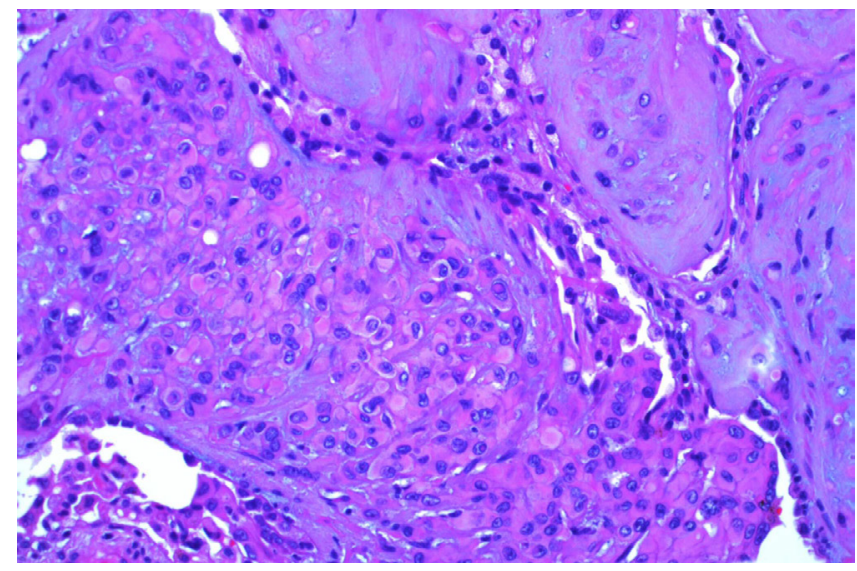

Figure 8: Epithelioid variable-sized cells with oval nucleus in Hyaline stroma $H \& E \times 20$.

A chest CT scan performed 4 months post operatively showed no evolution in the size or the number of the remaining nodules. Five months after surgery, the patient's clinical status is unchanged and she is not under any specific treatment protocol. A watchful observation has been proposed.

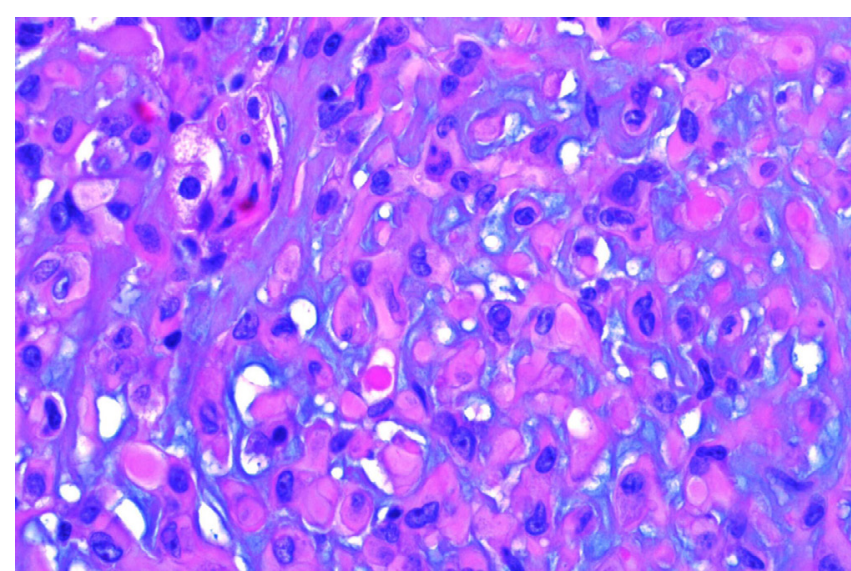

Figure 9: Eosinophilic cytoplasm and intracytoplasmic Vacuoles in tumour cells H\&E $x 40$.

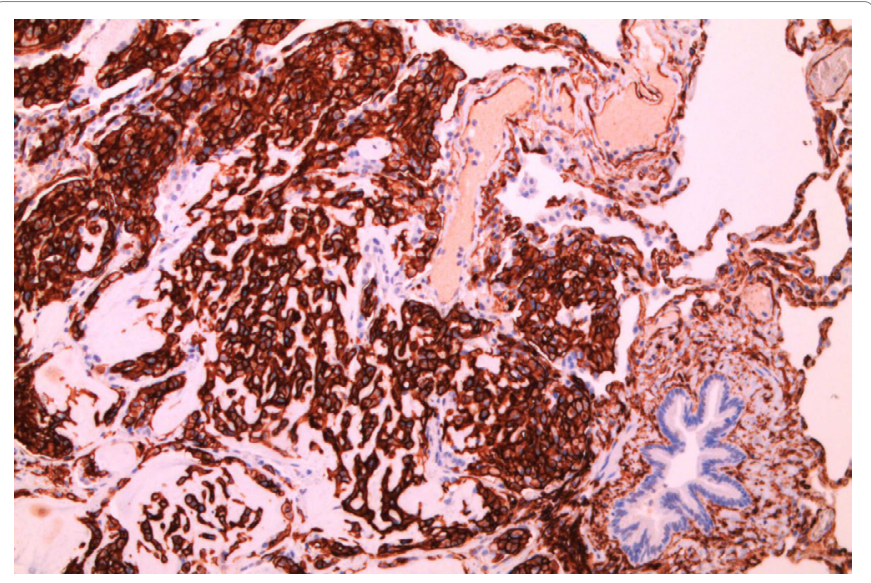

Figure 10: The tumour cells show cytoplasmic immunoreactivity for cd34 x40.

\section{Discussion}

Histologically, EHE presents a number of non-specific characteristics such as epithelioid cells with abundant eosinophilic cytoplasm in a fibromyxoid stroma and intracytoplasmic vacuoles. However, these characteristics may also be found in primary adenocarcinomas, mesotheliomas and B-cell lymphomas therefore highlighting the importance of immunohistochemical analyses for endothelial markers $[5,6]$. The aetiology of EHE is still unknown, though in its 2015 Classification of Tumours of the Lung, Pleura, Thymus and Heart, the WHO recognises the importance of a translocation involving the WWTR1 gene on chromosome $3 \mathrm{q} 25$ and the CAMTA1 gene on chromosome 1p36.23. Despite both genes were previously known to play key roles in oncogenesis, EHE is the first case in which both these genes are involved in a recurrent chromosomal translocation. This is of particular interest as CAMTA 1 and WWTR1 rearrangements are absent in epitheliod hemangiomas-benign tumours frequently misdiagnosed as EHE [7-9]. Furthermore, the WWTR1/CAMTA1 gene fusion could represent a target for the development of specific drugs. Interestingly, a 2011 report suggests a possible causal relationship between chronic Bartonella infection and the development of EHE. This is likely due to Bartonella's capability of inducing vascular endothelial growth factor-mediated vasoproliferation and long lasting intraerythrocytic and intraendothelial infections [10]. 
Clinical presentation is heterogenous with the majority of patients being asymptomatic at the time of diagnosis. Others present non-specific symptoms such as chest pain, dyspnea, cough, fatigue, hemoptysis and weight loss $[11,12]$. A diagnosis is usually made following initial abnormal findings in chest $\mathrm{x}$-rays during routine health examinations. Most frequently, P-EHE appears as multiple perivascular nodules with limited growth on serial chest $\mathrm{x}$-rays or CT scans [13-15]. The absence of growth in serial CT scans is a useful indicator in excluding differential diagnoses such as hematogenous metastases, pulmonary arteriovenous malformations, granulomatous infections and granulomatous diseases. Typically, nodules are bilateral and vary in size between less than $1 \mathrm{~cm}$ and up to $2 \mathrm{~cm}$ and are generally located in the inferior lobes of the lungs, in proximity to small and medium sized vessels and bronchi. However, in up to $20 \%$ of cases, P-EHE may appear as a solitary nodule [16].

In the presence of secondary lesions, bone metastases appear as osteolytic lesions with homogenous contrast enhancement and joint invasion. Lesions are usually found in cortical or medullary bone with cortical disruption and extension into soft tissue.

Magnetic resonance imaging shows no specific pattern of signal intensity. Low to intermediate signal intensity on T1-weighted images and high signal intensity on T2-weighted images with a homogenous enhancement with contrast medium are the most common signs of P-EHE [17].

Positron emission tomography (PET) as well as bone scintigraphy can be used in the assessment of secondary lesions although Cazzuffi et al. reported a case of a PET negative P-EHE [18]. Currently, there is still no typical biological or clinical marker for P-EHE. Blood tests are usually normal unless the liver is involved, in which case an abnormal levels of white blood cell count, serum alkaline phosphatase, aspartate aminotransferase, gammaglutamyl transferase, amylase and lipase may be seen [19].

To our knowledge, there are only 2 cases of P-EHE in the literature that have been diagnosed through a trans bronchial biopsy. The majority of cases were diagnosed through biopsies obtained via openlung or thoracoscopic procedures, as was the case with our patient. Globally, the prevalence of EHE is estimated to be of less than 1 in a million cases with approximately 248 cases of P-EHE reported in literature [20].

According to the international Hemangioendothelioma, Epithelioid Hemangioendothelioma and Related Vascular Disorders (HEARD) registry - an online database with data from 206 patientsthe most frequent localisations of EHE are the lung and liver with more than $60 \%$ of cases having a single organ presentation.

In two studies that reviewed 108 patients, the authors found that the average age of patients suffering from EHE was 40.1+/- 17.3 years with $73 \%$ of patients being female. $49.5 \%$ of patients were asymptomatic whilst the most common symptoms were dyspnea with cough $(18.3 \%$ of cases), chest pain (16\%), hemoptysis and weight loss (6.5\%) [11,21].

Reports on survival vary greatly. Three separate studies have shown a mean survival of 4.6 years with a range of 6 months to 24 years. Lau et al. found that the 5 year overall survival is $73 \%$ whilst mortality for lung disease after a 4 year follow up was reported to be $65 \%$ Survival in asymptomatic patients was 180 months [20].

Although no prognostic factors have been defined, common consensus is that pulmonary symptoms such as pleural hemorrhagic effusions, hemoptysis, hilar metastases and liver involvement are the worst prognostic factors, with an average survival of 2.2 years [17]. Surprisingly, the presence of single metastases at the time of diagnosis does not translate into a reduced survival. Furthermore, synchronous lesions of the skeleton and abdominal organs have no influence on survival if they remain localised and confined. On the other hand, skeletal involvement of three or more bones and the presences of ascites are to be considered as negative prognostic factors [22]. Independent predictors of survival are the presence of pulmonary lesions and a pleural effusion, with other significant risk factors being age $>54$, male gender, the presence of a cough, chest pain, multiple unilateral nodules, metastases to more than one site and lymph node metastases. Clinically P-EHE prognosis appears to be linked to vascular aggressiveness [12].

At this time, there is no approved standardised treatment protocol. Therapeutic options range from regular follow-ups with no active therapy in asymptomatic patients, surgical resections as well as combination chemotherapy [22-24]. Authors agree that surgery should be performed when possible; that is in cases of single pulmonary nodules or unilateral multiple nodules. In cases where a successful curative resection has been possible, good outcomes have been achieved.

Wedge resections offer similar survival outcomes as do anatomic resections and although the prognostic value of lymph node invasion is still unclear, hilar lymph node resection should be systematically proposed. Cases of bilateral nodules have been treated using interferon2 a most likely because of its antiangiogenic activity [17].

The role of chemotherapy in the treatment of P-EHE has proven to be beneficial in only a small number of cases with good partial responses when treated with carboplatin, paclitaxel, bevacizumab (a monoclonal antibody that blocks human Vascular Endothelial Growth Factor- $\alpha$ ), thalidomide and $a$-interferon. Bo Ye et al. described three cases of P-EHE with bilateral intrapulmonary masses which showed a partial response to combination chemotherapy with carboplatin, packetaxel, evacizumab or Endostar ${ }^{\star}$, a recombinant human endostatin. Although these regimens were unable to stop the progression of the disease, two of the three cases showed a stabilisation of the disease with a marked clinical improvement. Furthermore, angiogenesis inhibition may play a role in metastatic EHE by inhibiting angiogenesis through the use of bevacizumab [24].

There is also a role for the use of steroid modulators, as neoplastic cells express glucocorticoid receptors as well as the enzyme 11B-hydroxysteroid dehydrogenase that is involved in the synthesis of steroids [25]. In cases of pleural EHE, Pinet et al. reported a case resulting in complete remission following treatment with carboplatin associated with etoposide [26]. Radiation therapy has no indication in P-EHE due to its slow growth rate and radiobiological characteristics. However, 4-week protocols have been defined for extra pulmonary cases of EHE [25]. A possible future treatment option if the causal association with Bartonella where to be confirmed, could be to eradicate the bacterial infection thus interrupting the angiogenic and proliferative cell signals induced by Bartonella with the aim to slow tumour progression and improve patient outcomes [11].

\section{Conclusion}

P-EHE is a rare tumour with a wide array of clinical manifestations, biological evolution and outcomes.

Further research is required to correlate the various radiological findings to the molecular and genetic characteristics that have been identified until now, in order to assess their prognostic value and devise standardised treatment protocols. 
Citation: Ambrosetti T, Sgardello Sébastian D, Thomopoulos T, Koliakos E, StranoF, etal. (2018)Primary Pulmonary Epithelioid Hemangioendothelioma: A Case Report and Literature Review. Lung Dis Treat 4: 127. doi:10.4172/2472-1018.1000127

\section{References}

1. Weiss W, Ishak KG, Dail DH, Sweet DE, Enzinger FM (1986) Epithelioid hemangioendothelioma and related lesions. Semin Diagn Pathol 3: 259-287.

2. Dail DH, Liebow AA, Gmelich JT (1983) Intravascular, bronchiolar, and alveolar tumor of the lung (IVBAT): An analysis of twenty cases of a peculiar sclerosing endothelial tumor. Cancer 51: 452-464.

3. Mertens F, Unni K, Fletcher CDM (2002) World Health Organization classification of tumors. Pathology and genetics. Tumors of soft tissue and bone Lyon: IRAC Press PP: 155.

4. Corrin B, Manners B, Weaver L (1979) Histogenesis of the so-called intravascular bronchioloalveolar tumor. J Pathol 123: 163-167.

5. Flucke U, Vogels RJ, De Saint Aubain Somerhausen N (2014) Epithelioid Hemangioendothelioma: clinicopathologic, immunhistochemical, and molecular genetic analysis of 39 cases. Diagnostic Pathology 9: p131.

6. Palfoldi R, Radacs M, Csada E (2013) Pulmonary epithelioid haemangioendothelioma studies in vitro and in vivo: new diagnostic and treatment methods. In Vivo 27: 221-225.

7. Errani C, Zhang L, Sung YS (2011) A novel WWTR1-CAMTA1 gene fusion is a consistent abnormality in epithelioid hemangioendothelioma of different anatomic sites. Genes Chromosomes and Cancer 50: 644-653.

8. Anderson T, Zhang L, Hameed M (2015) Thoracic epithelioid malignant vascular tumors: a clinicopathologic study of 52 cases with emphasis on pathologic grading and molecular studies of WWTR1-CAMTA1 fusions. The Am J Surg Path 39: 132-139.

9. Travis WD, Brambilla E, Burke AP (2015) WHO Classification of Tumours of the Lung, Pleura, Thymus and Heart. WHO/IARC Classification of Tumours 7.

10. Mascarelli PE, Iredell JR, Maggi RG (2011) Bartonella species bacteremia in two patients with epithelioid Hemangioendothelioma. J Clinic Micro 49: 40064012.

11. Amin RM, Hiroshima K, Kokubo T (2006) Risk factors and independent predictors of survival in patients with pulmonary epithelioid hemangioendothelioma. Review of the literature and a case report. Respirology 11: 818-825.

12. Kitaichi M, NagaiS, Dail DH(1998)Pulmonary epithelioid hemangioendothelioma in 21 patients, including three with partial spontaneous regression. Eur Respir J 12: 89-96.
13. Kim EY, Kim TS, Han J (2011) Thoracic epithelioid hemangioendothelioma: Imaging and pathologic features. Acta Radiologica 52: 161-166.

14. Sakamoto N, Adachi S, Monzawa S (2005) High resolution CT findings of pulmonary epithelioid hemangioendothelioma: Unusual manifestations in 2 cases. J Thorac Imag 20: 236-238.

15. Luburich P, Ayuso MC, Picado C (1994) CT of pulmonary epithelioid hemangioendothelioma. J Comp Ass Tomo 18: 562-565.

16. Bagan $P$, Hassan M, Riquet $M$ (2006) Prognostic factors and surgical indications of pulmonary epithelioid hemangioendothelioma: a review of the literature. Ann Thorac Surg 82: 2010-2013.

17. Larochelle O, Périgny $M$, Giguère $C$ (2006) Best Cases from the AFIPepithelioid hemangioendothelioma of bone. Radio Graphics 26: 265-270.

18. Cazzuffi R, Calia N, Ravenna F (2011) Primary Pulmonary Epithelioid Hemangioendothelioma: A Rare Cause of PET-Negative Pulmonary Nodules. Case Reports in Medicine.

19. Kim SJ, Kim YC (2011) Unusual extrahepatic metastasis to the soft tissue of the left cervical neck area from hepatic epithelioid hemangioendothelioma. Hepatology 54: 1480-1481.

20. Lau K, Massad M, Weinberg G (2011) Clinical patterns and outcome in epithelioid hemangioendothelioma with or without pulmonary involvement. Chest 140: 1312-1318.

21. Schattenberg T, Kam R, Klopp M (2008) Pulmonary epithelioid hemangioendothelioma: Report of three cases. Surg Today 38: 844-849.

22. SardaroA, BardosciaL, PetruzzelliMF(2014)Epithelioid hemangioendothelioma: an overview and update on a rare vascular tumor. Oncol Rev 8: p259.

23. Ye B, Li W, Feng J (2013) Treatment of pulmonary epithelioid hemangioendothelioma with combination chemotherapy: Report of three cases and review of the literature. Oncology Letters 5: 1491-1496.

24. Mesquita RD, Sousa M, Trinidad C (2017) New Insights about Pulmonary Epithelioid Hemangioendothelioma: Review of the Literature and Two Case Reports. Case Reports in Rad 5: 972-940

25. Kumazawa Y, Maeda K, Ito M (2002) Expression of glucocorticoid receptor and $11 \beta$ hydroxysteroid dehydrogenase in a case of pulmonary epithelioid haemangioendothelioma. Molecular Path 55: 61-64.

26. Pinet C, Magnan A, Vervloet D (1999) Aggressive form of pleural epithelioid hemangioendothelioma: complete response after chemotherapy. Eur Respir J 14: $237-238$ 